\title{
Deep Learning for Multisensor Image Resolution Enhancement
}

\author{
Charles B. Collins \\ Information Technology and Systems \\ Center \\ University of Alabama in Huntsville \\ Huntsville, AL 35899 \\ Charles.B.Collins@uah.edu
}

\author{
John M. Beck \\ Information Technology and Systems \\ Center \\ University of Alabama in Huntsville \\ Huntsville, AL 35899 \\ John.Beck@uah.edu
}

\author{
Susan M. Bridges \\ Information Technology and Systems \\ Center \\ University of Alabama in Huntsville \\ Huntsville, AL 35899 \\ Susan.M.Bridges@uah.edu
}

\author{
John A. Rushing \\ Information Technology and Systems \\ Center \\ University of Alabama in Huntsville \\ Huntsville, AL 34899 \\ John.Rushing@uah.edu
}

\begin{abstract}
${ }^{1}$
We describe a deep learning convolutional neural network (CNN) for enhancing low resolution multispectral satellite imagery without the use of a panchromatic image. For training, low resolution images are used as input and corresponding high resolution images are used as the target output (label). The CNN learns to automatically extract hierarchical features that can be used to enhance low resolution imagery. The trained network can then be effectively used for super-resolution enhancement of low resolution multispectral images where no corresponding high resolution image is available. The $\mathrm{CNN}$ enhances all four spectral bands of the low resolution image simultaneously and adjusts pixel values of the low resolution to match the dynamic range of the high resolution image. The $\mathrm{CNN}$ yields higher quality images than standard image resampling methods.
\end{abstract}

\section{CCS CONCEPTS}

- Computing methodologies $\rightarrow$ Machine learning approaches • Computing methodologies $\rightarrow$ Neural networks

- Applied computing $\rightarrow$ Earth and atmospheric sciences

\section{KEYWORDS}

Deep Learning, Convolutional Neural Networks, Remote Sensing, Image Resolution, Super Resolution, Multispectral

ACM Reference format:

C.B. Collins, J.M. Beck, S.M. Bridges, J.A. Rushing, S.J. Graves, 2017. In GeoAI'17: $1^{\text {st }}$ ACM SIGSPATIAL Workshop on Artificial

\footnotetext{
${ }^{1}$ Permission to make digital or hard copies of all or part of this work for personal or classroom use is granted without fee provided that copies are not made or distributed for profit or commercial advantage and that copies bear this notice and the full citation on the first page. Copyrights for components of this work owned by others than ACM must be honored. Abstracting with credit is permitted. To copy otherwise, or republish, to post on servers or to redistribute to lists, requires prior specific permission and/or a fee.Request permissions from Permissions@acm.org.

GeoAl'17, November 7-10, 2017, Los Angeles Area, CA, USA

(C) 2017 Association for Computing Machinery.

ACM ISBN 978-1-4503-5498-1/17/11 \$15.00

https://doi.org/10.1145/3149808.3149815
}

\author{
Sara J. Graves \\ Information Technology and Systems \\ Center \\ University of Alabama in Huntsville \\ Huntsville, AL 35899 \\ Sara.Graves@uah.edu
}

Intelligence and Deep Learning for Geographic Knowledge Discovery, November 7-10, Los Angeles Area, CA, USA, 8 pages. https://doi.org/10.1145/3149808.3149815

\section{INTRODUCTION}

Large quantities of remotely sensed data are available for today's researchers to assist in monitoring earth's land surfaces, estuaries, coastal areas, and oceans. At least seventy different operational satellites equipped with various sensors provide data with different spatial, spectral, and temporal resolutions [1]. Geospatial events such as landslides, earthquakes, floods, etc. occur across a variety of spatial and temporal scales and rapid detection and analysis requires the best spatial, spectral, and temporal resolutions available at any one time. When high resolution data is not available, a rapid and accurate multisensor image spatial resolution enhancement method is needed for super-resolution of low spatial resolution images without compromising spectral information. This process enables more effective, efficient, and productive use of the available spatial resolution multispectral image (MSI) data.

Over the past few years, Deep Learning Convolutional Neural Networks (CNNs) have consistently outperformed traditional machine learning and other algorithmic approaches for many image-processing tasks [2, 3] including image segmentation, object identification, image classification, image understanding, etc. $[2,4-10]$. A recent flurry of research in photographic image super-resolution via deep learning [11-13] and the use of similar techniques for pan sharpening [14], demonstrate the potential for deep learning as an effective method for enhancement of remotely sensed multispectral images.

The work reported in this paper takes advantage of the availability of concurrently collected low resolution images from the Advanced Wide Field Sensor (AWiFS) sensors and high resolution images from the Linear Imaging Self Scanner (LISS-III) sensors aboard the Indian Space Research Organisation's (ISRO) Resourcesat- 1 and -2 missions. We use this unique data set to demonstrate that a $\mathrm{CNN}$ can learn features from matching pairs of low and high resolution satellite-based remotely sensed imagery to automate spatial resolution enhancement of low 
resolution multisensor data without the use of a panchromatic image. The CNN training and test data are taken from coincident regions of the LISS-III and AWiFS data. During training, the network is shown corresponding low resolution AWiFS (input) and LISS-III high resolution (output target) image patches and learns how to recognize structure in the low resolution patches such as edges, masses of vegetation, rivers, roads, etc. and to appropriately interpolate this structure during the enhancement process to produce a multispectral image that more closely resembles the high resolution image. The features learned by the $\mathrm{CNN}$ are general and the trained network can subsequently be used to enhance the spatial resolution of AWiFS images where no corresponding LISS-III image is available.

\section{BACKGROUND}

\subsection{Deep Learning and Convolutional Neural Networks}

Convolutional neural networks differ from more traditional neural networks in several major related respects. In traditional neural networks every node in a layer is connected to every node in the next layer. This means that as the number of layers grows, the number of weights to be learned increases dramatically and makes the learning process very difficult. Thus, most traditional neural networks have only a few layers. Convolutional networks take advantage of the spatial coherence of data. Each convolutional layer consists of a number of filters and units in each layer are connected to a limited number of adjacent units in the next layer. Limiting the number of connections makes it possible to use deeper networks, where each layer learns hierarchical abstractions of the input. The maintenance of spatial coherence also facilitates end-to-end mapping of an input image to an enhanced output image.

\subsection{Methods for Increasing Spatial Resolution}

Combining data from two or more sensors to increase spatial resolution has been an active area of research for many years [15-21]. Several studies have demonstrated methods for combining MSI data with panchromatic [15], microwave [17], and synthetic aperture radar (SAR) data [18] with good success. Many different algorithms and techniques have been tested including Intensity-Hue-Saturation (IHS) [19, 22], Principal Component Analysis (PCA) [18, 20, 23], Discrete Wavelet Transformation (DWT) [21], Support Vector Regression (SVR) $[13,24]$, and various combinations of these methods [22, 25, 26].

Most of these methods use a higher-spatial resolution (HSR) panchromatic image to improve the spatial resolution of the MSI data $[15,21,25,27]$. These techniques often display high spectral distortions because the panchromatic and MSI bands of data do not overlap entirely in the electromagnetic spectrum.

\subsection{CNN-Based Image Super-Resolution}

In a ground-breaking study, Dong, et al. [11] demonstrated that deep CNNs can be used to directly learn an end-to-end mapping between a low spatial-resolution photographic image (LSR) and its corresponding high spatial resolution (HSR) image for image super-resolution of photographs. Both the original researchers [28] and several other groups [29-31] have since published a variety of improvements to this approach for image superresolution to enhance both learning speed and quality of the resulting image. For example, ideas from this have been used to investigate the use of image super-resolution and CNNs with satellite based remotely sensed imagery to upscale the resolution of LSR images with HSR images [12, 32, 33] using panchromatic sharpening. Researchers use a variety of methods to combine the pan image with one or more channels of the multispectral image.

The successful application of CNNs is critically dependent on the availability of large labeled training datasets-in this case corresponding LSR and HSR images over the same area, at the same time, with the same spectral properties. Research groups working with pan sharpening of remotely sensed data have generated training data by downsampling high resolution multispectral images $[12,32]$ to yield corresponding low resolution images. These low resolution images together with corresponding panchromatic images are used as input to a $\mathrm{CNN}$ trained to regenerate the original high resolution image. Researchers in super-resolution of photographic images use a similar approach for generating matching low and high resolution images for network training $[11,28,29]$. The trained network can then be used for enhancement of previously unseen low resolution images.

The goal of our research is develop to a super resolution method to enhance the spatial resolution of low-resolution remotely sensed images without the use of a corresponding highresolution panchromatic image or any other high resolution image. Unlike previous work in super resolution, the low resolution images used for training are not derived from the corresponding high resolution image, but are native images collected independently from a separate sensor with the same spectral bands taken at the same time.

\subsection{Resourcesat-1 and -2 Satellite Imagery}

Our research takes advantage of unique sensors on the Indian Space Research Organisation's (ISRO) Resourcesat-1 and 2 missions. These satellites' two sensors, the Advanced Wide Field Sensor (AWiFS) and the Linear Image Self Scanner (LISS-III), collect data with the same four spectral bands but at different resolutions due to differences in the swath width [26].

In simultaneous acquisition, the LISS-III sensor's $140 \mathrm{~km}$ swath overlaps the center of the AWiFS $740 \mathrm{~km}$ swath [13] providing a unique opportunity to use the coincident images with the same spectral bands but different spatial resolutions to build a training set for Deep Learning. Regions where AWiFS imagery is available, but LISS-III imagery is not can then be used to demonstrate that the $\mathrm{CNN}$ is learning general features that can be used to enhance LSR images with no pan or HSR image.

Resourcesat-1 and -2 satellites operate in a sun-synchronous orbit at an altitude of $817 \mathrm{~km}$. The satellites take 101.35 minutes 
to complete one revolution around the earth and complete about 14 orbits per day. The entire earth is covered by 341 orbits during a 24-day cycle $[34,35]$. The LISS-3 and AWiFS instruments on the Resourcesat- 1 and -2 satellites acquire four spectral bands ranging from the Visible and Near-Infrared (VNIR) to Shortwave Infrared (SWIR) wavelengths. The LISS-3 sensor covers a $140-\mathrm{km}$ orbital swath with a product spatial resolution of 24 meters and a 24 -day repeat cycle. The AWiFS sensor covers a $740-\mathrm{km}$ orbital swath at a resolution of 56 meters and provides a 5-day repeat cycle [34, 35]. The characteristics of the LISS-III and AWiFS sensors are given in Table 1.

Table 1. Characteristics of LISS-III and AWiFS Sensors

\begin{tabular}{|c|c|c|}
\hline & LISS III & $A W i F S$ \\
\hline \multirow{4}{*}{$\begin{array}{l}\text { Four Multispectral } \\
\text { Bands and Wavelength } \\
\text { Ranges }(\mu \mathrm{m})\end{array}$} & $\begin{array}{l}\text { Band 2: } 0.52 \quad- \\
0.59 \text { (Green) }\end{array}$ & $\begin{array}{l}\text { Band 2: } 0.52-0.59 \\
\text { (Green) }\end{array}$ \\
\hline & $\begin{array}{l}\text { Band 3: } 0.62 \quad- \\
0.68 \text { (Red) }\end{array}$ & $\begin{array}{l}\text { Band 3: } 0.62-0.68 \\
\text { (Red) }\end{array}$ \\
\hline & $\begin{array}{l}\text { Band 4: } 0.77 \text { - } \\
0.86 \text { (NIR) }\end{array}$ & $\begin{array}{l}\text { Band 4: } 0.77-0.86 \\
\text { (NIR) }\end{array}$ \\
\hline & $\begin{array}{l}\text { Band 5: } 1.55 \text { - } \\
1.70 \text { (SWIR) }\end{array}$ & $\begin{array}{l}\text { Band 5: } 1.55-1.70 \\
\text { (SWIR) }\end{array}$ \\
\hline Spatial Resolution (m) & 24 & 56 \\
\hline $\begin{array}{l}\text { Temporal Resolution } \\
\text { (Revisit Days) }\end{array}$ & 24 & 5 \\
\hline Swath Width $(\mathrm{km})$ & 140 & 740 \\
\hline
\end{tabular}

Previous work by others has taken advantage of these coincident images to enhance the spatial resolution of the AWiFS images by using sub pixel relationships, contourlet coefficients, and SVMs $[13,24,26]$. The reported results using SVM's are promising but are limited to a case where both the training and test data are sampled from a single image. In general, SVM's require custom feature extraction and are effective with very small training sets with few outliers. In the research reported in this paper, a CNN is trained to learn features from a large set of corresponding images and is tested on a completely different set of images. Our Deep Learning CNN requires little data preprocessing and can effectively learn hierarchies of features from large training datasets that provide general solutions to difficult image processing problems with higher accuracy.

\subsection{Contributions}

The major contributions of this research are the following: 1) we demonstrate that convolutional neural networks can be effectively used to enhance the resolution of remotely sensed multispectral low resolution images without the use of high resolution panchromatic images; 2) the network is able to learn a mapping between independently collected input and output images pairs. The structural features learned by the network are general and can then be used to enhance low resolution images not used for training and those where no high resolution image is available; 3) all channels of the images are enhanced simultaneously and the dynamic range of pixel values is adjusted to match that of the high resolution images to facilitate fusion of enhanced low resolution images with high resolution images.

\section{CONVOLUTIONAL NETWORK}

\subsection{Convolutional Architecture}

CNNs are commonly used for a wide variety of image classification tasks. In these applications, the networks automatically learn hierarchical features that allow them to recognize characteristics of the items being classified and to output a class label. In contrast, CNNs used for super-resolution learn hierarchical features that allow them to output an enhanced interpolated multispectral image [11, 28, 29]-a much more complex output than for CNN's trained for classification tasks where the output is typically a single class. The ability of CNNs to maintain spatial coherence of the inputs is important for both classification and super-resolution. For super-resolution tasks, the network must also maintain the spatial dimensions of the input. The task of generating each pixel in the output is a

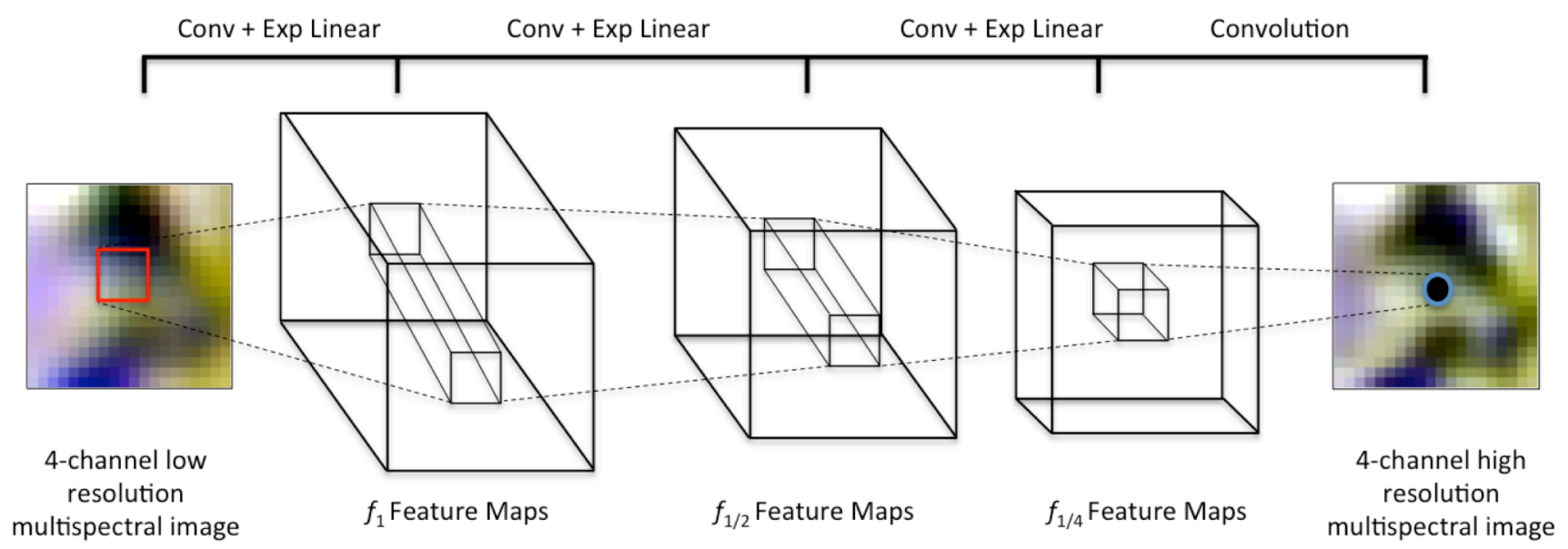

Figure 1. Convolutional neural network architecture. 
regression task rather than a classification task. The network learns a set of features that make use of information embedded in neighboring pixels to generate an interpolated value for each pixel in all four spectral bands of the image simultaneously thus generating an entire multispectral image.

The network we use for the enhancement of satellite imagery is based on the ground-breaking super-resolution convolutional neural network (SRCNN) proposed by Dong et al. [11]. Like SRCNN, our network is relatively shallow and purely convolutional as illustrated in Figure 1. The network consists of three to five convolutional layers where the number of filters decreases by one-half as the depth of the network increases. With the exception of the output layer, each layer applies an activation function.

One problem with the SRCNN approach is that the network output is a subimage of the input image, with the size of the output being dependent upon the filter sizes used by its constituent convolutional layers. This is due to the feature map decreasing in size through the network. To avoid this effect, we employ the technique developed by Kim et al. [29] of using zero padding of the input and then applying $3 \times 3$ filters, resulting in a feature map with the same dimensions as the input. Hence, the network produces a super-resolved image corresponding to the entire input image with no loss of border pixels.

\subsection{Activation Functions}

Rectified linear units (ReLUs) typical of convolutional networks were initially used as the activation function for the convolutional layers in our network [11, 28, 29]. However, in early experiments ReLUs would "die" and only output zeros. To remedy this issue, the exponential linear unit (ELU) [36] was adopted as the activation function in all non-output layers. The ELU function avoids entering into a "dead" state by including negative values in its range. The magnitude of the introduced negative values can be tuned through a hyper-parameter.

For the ELU, activation of each non-output layer can be expressed as a function $\mathrm{F}$ and the output layer as a function $\mathrm{G}$ :

$$
\begin{gathered}
F(X)=\max (0, C * X+b)+\alpha\left(e^{\min (0, C * X+b)}-1\right) \\
G(X)=C * X+b
\end{gathered}
$$

where $X$ is the input image or feature map, $C$ is a set of convolutional filters, * denotes convolution, $b$ is the bias vector,

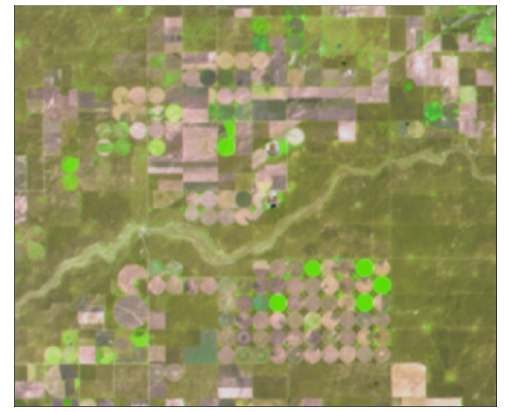

and $\alpha$ is the tunable, positive hyper-parameter of the ELU function.

\subsection{Quality Assessment}

Many different methods for quality assessment of image interpolation, super-resolution, or pan sharpening methods have been developed [37]. Two of the most commonly used methods are Peak Signal to Noise Ratio (PSNR) [38] and the structural similarity (SSIM) index. For this project, PSNR and SSIM were calculated using the scikit-image Python library. The peak signal to noise ratio of an image $\mathrm{X}$ with relation to the original or target image $\mathrm{Y}$ is as follows:

$$
\operatorname{PSNR}(X, Y)=10 \cdot \log _{10} \frac{L^{2}}{\operatorname{MSE}(X, Y)}
$$

where $\mathrm{L}$ is the maximum pixel value and MSE is the mean square error between image $\mathrm{X}$ and image $\mathrm{Y}$. We use $\mathrm{L}=1023$ since the target high resolution image uses a 10-bit unsigned integer range. SSIM is calculated using the parameters suggested by Wang et al. [37] as follows:

$$
\operatorname{SSIM}(X, Y)=\frac{\left(2 \mu_{X} \mu_{Y}+C_{1}\right) \cdot\left(2 \sigma_{X Y}+C_{2}\right)}{\left(\mu_{X}^{2}+\mu_{Y}^{2}+C_{1}\right) \cdot\left(\sigma_{X}^{2}+\sigma_{Y}^{2}+C_{2}\right)}
$$

where $\mu_{X}$ and $\mu_{Y}$ are the mean pixel values of images $\mathrm{X}$ and $\mathrm{Y}$, $\sigma_{X}^{2}$ and $\sigma_{Y}^{2}$ are the variance of pixel values in images $\mathrm{X}$ and $\mathrm{Y}$, $\sigma_{X Y}$ is the covariance of images $\mathrm{X}$ and $\mathrm{Y}$, and $C_{i}=k_{i} \cdot L$ where $k_{i}<1$ and $\mathrm{L}$ is the maximum pixel value. As with PSNR, we use $\mathrm{L}=1023$. The values $k_{1}=0.01$ and $k_{2}=0.03$ are used by default in scikit-image.

\section{Experimental Results}

\subsection{Data}

4.1.1 Data Acquisition. Resourcesat-1 and -2 data were acquired from the United States Geological Survey's (USGS's) EarthExplorer web based application located at https://earthexplorer.usgs.gov/. A series of images over the Midwestern portion of the United States of America (USA) were downloaded. Care was taken to select images with little or no cloud cover and that contained enough Global Land Survey (GLS) stations within the image to provide a Precision Terrain Correction (Level-1T) product. The Level-1T product provided a higher level of geometric correction. The LISS-3 products were referenced to the Universal Transverse Mercator/World Geodetic System 1984 (UTM/WGS84) projection and datum and the

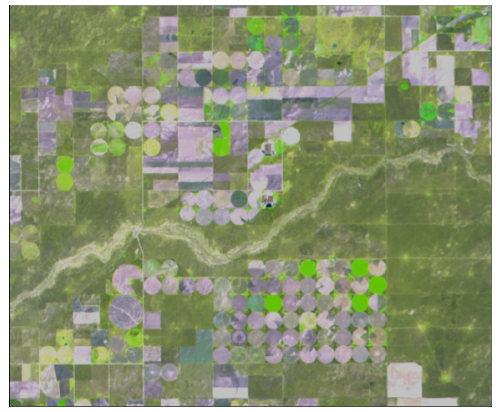

Figure 2. Comparison of low resolution AWiFS scene (left) and high resolution LISS-III scene (right). 
AWiFS products were referenced to the Lambert Conformal Conic/World Geodetic System 1984 (LCC/WGS84) projection and datum. The download package from the USGS included one Georeferenced Tagged Image File Format (GeoTIFF) file for each of the four spectral bands plus its corresponding metadata. It should be noted that the LISS-III data were provided as 10-bits per pixel data and the AWiFS data were distributed as 12-bit per pixel data. Figure 2 shows an example of corresponding LISS-III and AWiFS scenes.

4.1.2 Preparation of Training and Test Datsets. Satellite imagery presents notable challenges as a dataset. The raster arrays are too large to be used to efficiently train a neural network. Using an entire satellite image as input requires very small batch sizes due to memory constraints. A few preprocessing steps were needed to prepare suitable and practical input images for the network. Geospatially overlapping subimages were cropped from the two source images. The low resolution subimage was then scaled to the same resolution as the high resolution subimage using cubic resampling as implemented in GDAL [39]. Due to the impracticality of feeding a neural network such large images, the overlapping subimages were divided into patches of size 20x20 pixels. Images of this size are more reasonable for training a network and are also closer to images sizes used in the super resolution literature.

The resulting dataset of corresponding high and low resolution image patches consists of 76,440 input (low resolution patch) and corresponding target output (high resolution) pairs. Note that we use the term "target output" rather than "label" as is typical for classification. Sixty percent of the image pairs were randomly selected to form the training set for each experiment and the remaining $40 \%$ were used as the test set.

\subsection{Environment/Framework}

Our CNN was implemented in Python using Nervana Systems' Neon framework. All experiments were executed on an NVidia Tesla K20 GPU.

\subsection{Choice of Optimizer for Network Training}

Neural networks used for image enhancement traditionally use stochastic gradient descent with momentum (SGD+M) for optimization. Initial experiments were performed with momentum 0.9 , weight decay 0.001 , and learning rate $10^{-7}$. A learning rate of $10^{-7}$ was the largest at which consistent convergence would occur with our network. This resulted in a slow training process requiring thousands of epochs.

To speed up training, we used Adam as the optimizer in subsequent experiments. Adam is an adaptive optimization method proposed by Kingma and Ba [40]. We initially used the suggested default hyper-parameter settings from [40], $\alpha=0.001$, $\beta_{1=} 0.9, \beta_{2}=0.999$, and $\varepsilon=10^{-8}$. More stable convergence was attained after tuning $\varepsilon$ to 0.001 . The use of Adam for optimization substantially decreased number of training epochs and thus the amount time needed to train our network. The learning curves in Figure 3 illustrate the dramatic improvement
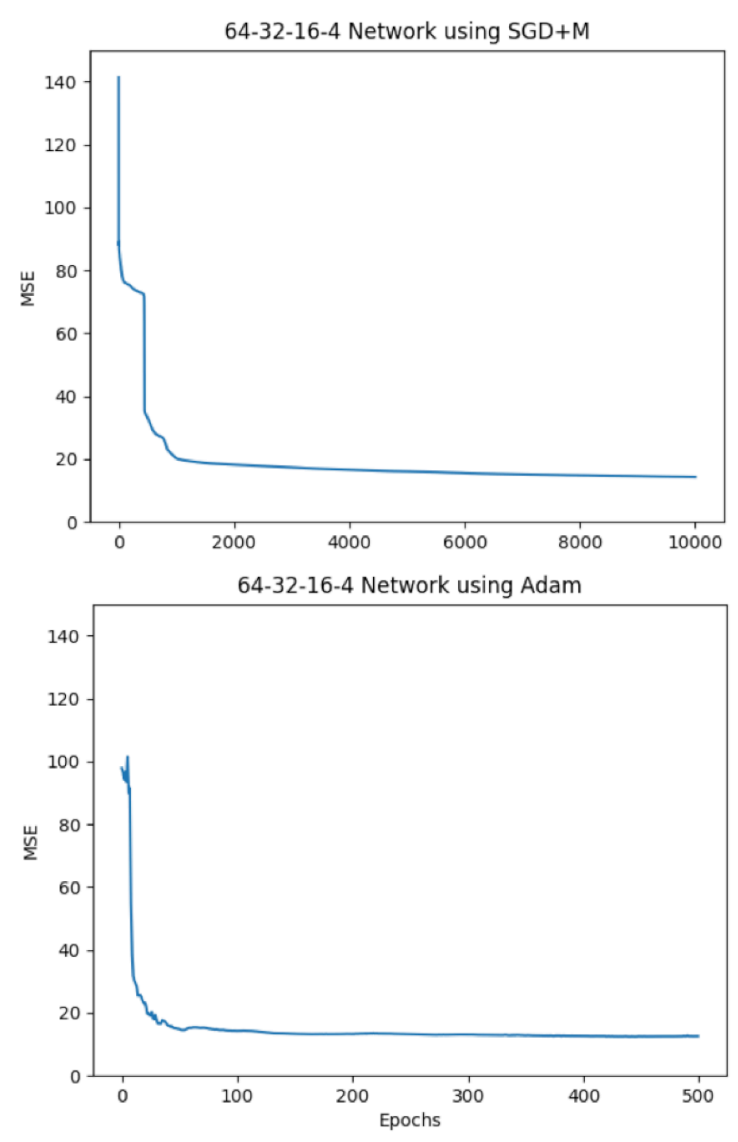

Figure 3. Comparison of convergence rates using SGD+M optimization (top) and Adam optimization (bottom). Note the difference of scale of the $\mathrm{x}$-axes.

in the rate of convergence when using Adam instead of SGD+M optimization where only the optimization method is varied. The network use for this comparison consists of four layers with 64, 32, 16, and 4 convolutional filters. Since Adam is an adaptive method, manual maintenance of a learning schedule is unnecessary.

\subsection{Dynamic Range of Pixel Values}

One challenge faced by our $\mathrm{CNN}$ is that the dynamic ranges for pixel values in the low resolution AWiFS image are based on 12bit values while those of the LISS-III image are based on 10-bit values. In previous image enhancement research using SVMs [13] with this data, the DN values for the high resolution data were normalized to the range of the low resolution data prior to processing. We have found that our network is able to learn how to automatically adapt the 12-bit $\mathrm{DN}$ values to a 10-bit range and thus avoid this preprocessing step.

It should be noted that due to the different DN ranges of the AWiFS imagery and LISS-III imagery, the DN values of an image interpolated from an AWiFS image must be converted to the DN range of LISS-III images in order to make a fair comparison [13]. 
Radiance values of the interpolated image are first computed, and then the equivalent LISS-III DN is calculated using those radiance values. The conversion applies the following equations as element-wise operations on the interpolated image's raster matrix:

$$
\begin{gathered}
\text { Radiance }=A W i F S D N \times \frac{\text { AWiFS Saturation Radiance }}{A W i F S \text { Maximum } D N} \\
\text { LISS DN }=\text { Radiance } \times \frac{\text { LISS Saturation Radiance }}{\text { LISS Maximum } D N}
\end{gathered}
$$

\subsection{Effect of Different Numbers of Layers and Filters}

Table 2 shows nine variations in network structure that were investigated. The number of layers and number of convolutional filters were varied while all other parameters were kept constant. Each network was trained for 500 epochs using Adam for network optimization and mean-squared error for its cost function. The subimages produced by the network were then merged to create a full satellite image which was compared to the target LISS III image using peak signal to noise ratio (PSNR) and structural similarity (SSIM) index for quality assessment as shown in Table 2.

Table 2 indicates that the network's learning capacity generally improves with a deeper and wider (i.e., more filters) structure. Qualitative comparison of the output images through inspection also shows marginal improvements in image detail for deeper and wider networks. However, network width has a substantial effect on execution time. Doubling the number of filters in the network increases execution time per epoch two- to three-fold.

\subsection{Example Images}

Figure 4 shows image segments illustrating the differences in the input original low resolution AWiFS image, the corresponding target high resolution LISS-III image, and the enhanced image output by the network. Note that neither the input low resolution or the target output image were part of the training set.
Table 2. Experimental results with different network structures. The numbers to the left designate the number of filters per layer of the neural network.

\begin{tabular}{|l|l|l|l|}
\hline $\begin{array}{l}\text { Network } \\
\text { Structure or } \\
\text { Interpolation } \\
\text { Method }\end{array}$ & PSNR & SSIM & $\begin{array}{l}\text { Mean } \\
\text { Training } \\
\text { Time per } \\
\text { Epoch (in } \\
\text { seconds) }\end{array}$ \\
\hline $32-16-4$ & 45.59 & 0.9827 & 2.50 \\
\hline $64-32-4$ & 46.10 & 0.9839 & 4.90 \\
\hline $128-64-4$ & 46.09 & 0.9838 & 14.83 \\
\hline $32-16-8-4$ & 45.86 & 0.9835 & 3.04 \\
\hline $64-32-16-4$ & 46.46 & 0.9850 & 5.78 \\
\hline $128-64-32-4$ & 46.69 & 0.9858 & 16.80 \\
\hline $32-16-8-4-4$ & 46.03 & 0.9837 & 3.52 \\
\hline $64-32-16-8-4$ & 46.49 & 0.9851 & 6.25 \\
\hline $128-64-32-16-4$ & 46.99 & 0.9862 & 17.73 \\
\hline
\end{tabular}

\subsection{Comparison to Other Methods}

Images produced by our network are notably more detailed than those produced by common interpolation methods implemented in GDAL [39] as shown in Table 3.

Table 3. Comparison of Image Quality Produced by CNN and Other Standard Methods.

\begin{tabular}{l|ll}
\hline $\begin{array}{l}\text { Network Structure or } \\
\text { Interpolation Method }\end{array}$ & PSNR & SSIM \\
\hline CNN (128-64-32-16-4) & 46.99 & 0.9862 \\
Nearest Neighbor & 32.39 & 0.9550 \\
Bilinear & 32.43 & 0.9576 \\
Cubic & 32.45 & 0.9593 \\
\hline
\end{tabular}

Thus, not only is the quality of images produced by the CNN substantially higher than the quality of images produced by other interpolation methods, the AWiFS image is also automatically converted to the same DNN range as the LISS-III

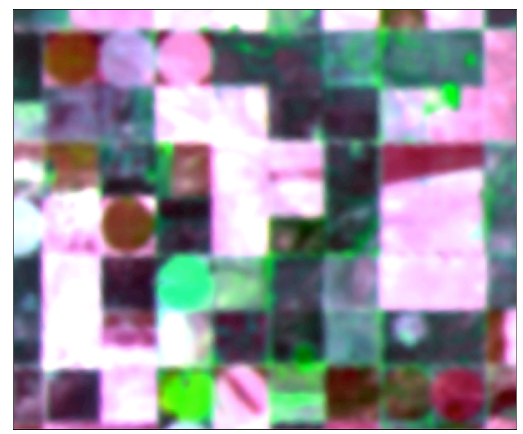

a.

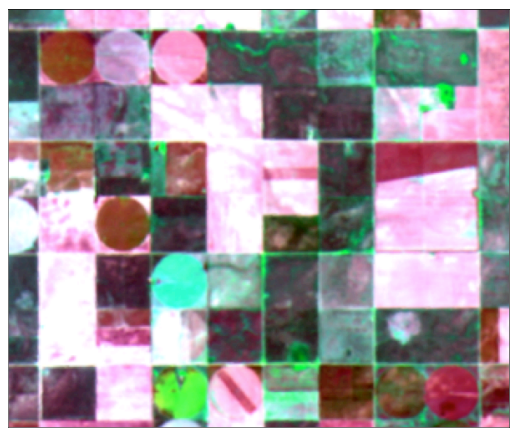

b.

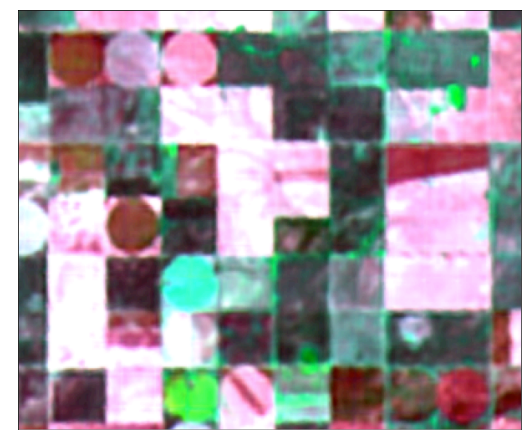

c.

Figure 4. Image snapshots illustrating differences in a) input low resolution AWiFS image upsampled with cubic interpolation, b) the target high resolution LISS-III image, and c) the output enhanced deep learning neural network image. 
images.

\subsection{Results with No High Resolution Image}

In the results we have shown thus far, concurrently collected high and low resolution imagery was used to provide training/test datasets and to evaluate image quality compared to other methods.

In actual practice, the network is designed to be used to enhance low resolution imagery where no high resolution imagery is available. With the AWiFS and LISS-III imagery, for example, the low resolution AWiFS imagery can be enhanced as it is collected and merged with the concurrently collected high resolution LISS-III imagery that is available-providing the most up-to-date high resolution imagery in a timely manner. The fact that our CNN not only enhances the AWiFS images but also translates them into a data range compatible with the LISS-III imagery makes the fusion process straightforward.

In Figure 5, we show enhanced AWiFS scenes produced by the $\mathrm{CNN}$ for two areas where no corresponding high resolution image was available. The images are compared to an image upsampled using cubic interpolation. Note that the CNN was trained using image pairs from a region where both high and low resolution imagery is available, and the trained network was then used to enhance AWIFS scenes where no corresponding high resolution LISS-III imagery was available. Features such as the runway at the bottom left of the second set of images are
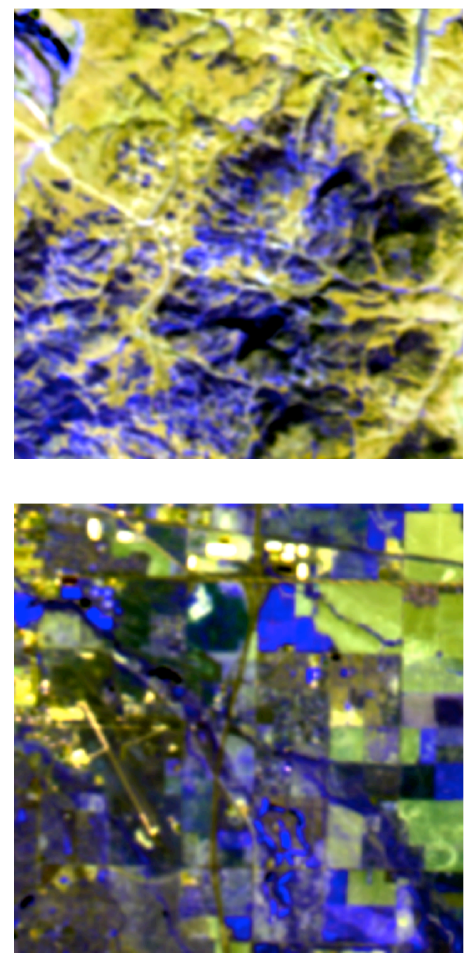

much crisper in the $\mathrm{CNN}$ generated picture.

\section{CONCLUSIONS AND FUTURE WORK}

The research described in this paper demonstrates that a $\mathrm{CNN}$ can be used as an end-to-end mapping method for enhancing low resolution images without the use of a panchromatic image. Unlike previous research using panchromatic images, the low resolution images in this research were not derived from the high resolution image, but are native low resolution images. High resolution imagery collected at the same time and place as the low resolution imagery is used to construct the training/test set. During the training process, the network automatically learns features needed to interpolate structural components of the low resolution image. Once the network is trained, the network can be used to enhance low resolution images where no high resolution imagery is available. The $\mathrm{CNN}$ also learns to automatically adjust the dynamic range of the low resolution images (AWiFS) to LISS-III dynamic range thus facilitating fusion of enhanced AWiFS images taken over a wide geographic area with LISS-III images taken simultaneously over more restricted ranges. Our $\mathrm{CNN}$ approach outperforms standard image interpolation techniques such as bilinear and cubic interpolation. The trained CNN could be implemented as part of an image processing pipeline for AWiFS and LISS-III imagery where the AWiFS imagery is routinely enhanced and fused with the concurrently collected higher resolution LISS-III imagery to
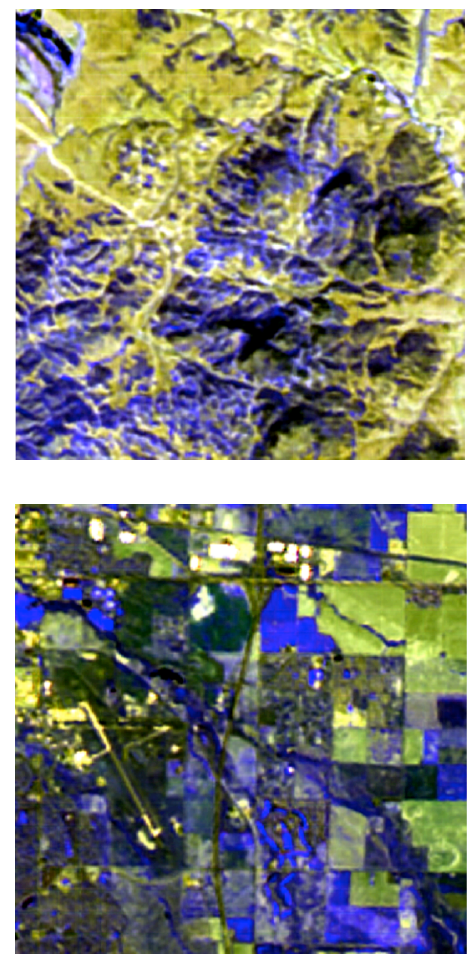

Figure 5. Comparison of AWiFS scenes enhanced by cubic interpolation (left) and by our CNN method (right) for a region where no corresponding high resolution images are available. 
expand the spatial and temporal extent of high resolution imagery.

In the experiments reported in this paper, we have used images from the Midwest US for both training and testing the CNN and for demonstrating its use with AWiFS data where no LISS-III data is available. If applied to a much larger geographic area such as North America, the training set would need to be expanded to contain image pairs from representative landscapes (urban, suburban, forest, lakes, mountains, small farms, etc.) and the network would probably need additional layers and filters to represent a wider variety of structural features. In the future we plan to investigate using the low resolution image directly for input without first interpolating to the same size as the high resolution image. Recent research has also shown the benefit of custom cost functions for super-resolution of photographs [31]. We plan to investigate development of such a cost function customized for remotely sensed multispectral images.

We hypothesize that the methods we have used for AWiFS and LISS-III imagery can be extended to enhance low resolution multispectral images for fusion with high resolution images where there is not only a mismatch in temporal and spatial resolution, but also in spectral bands such as with Landsat-7/8 and Sentinel-2 imagery.

\section{ACKNOWLEDGMENTS}

This work was supported by the Information Technology and Systems Center at the University of Alabama in Huntsville.

\section{REFERENCES}

[1] University of Twenty Faculty of Geo-Information and Earth Observation (ITC) ITC's database of satellites and sensors. 2017.

[2] LeCun, Y., Bengio, Y. and Hinton, G. Deep learning. Nature, 521, 7553 (2015), 436-444.

[3] Nielsen, M. A. Neural Networks and Deep Learning. Determination Press, 2015. [4] Badrinarayanan, V., Handa, A. and Cipolla, R. Segnet: A deep convolutional encoder-decoder architecture for robust semantic pixel-wise labelling. arXiv preprint arXiv:1505.07293 (2015).

[5] Basu, S., Ganguly, S., Mukhopadhyay, S., DiBiano, R., Karki, M. and Nemani, R. Deepsat: a learning framework for satellite imagery. In Proceedings of the Proceedings of the 23rd SIGSPATIAL International Conference on Advances in Geographic Information Systems (Seattle, WA, 2015). ACM.

[6] Farabet, C., Couprie, C., Najman, L. and LeCun, Y. Learning hierarchical features for scene labeling. IEEE transactions on pattern analysis and machine intelligence, 35 , 8 (2013), 1915-1929.

[7] Szegedy, C., Liu, W., Jia, Y., Sermanet, P., Reed, S., Anguelov, D., Erhan, D., Vanhoucke, V. and Rabinovich, A. Going deeper with convolutions. In Proceedings of the Proceedings of the IEEE Conference on Computer Vision and Pattern Recognition (Boston, MA, 2015).

[8] Tompson, J. J., Jain, A., LeCun, Y. and Bregler, C. Joint training of a convolutional network and a graphical model for human pose estimation. City, 2014 [9] Chen, S., Wang, H., Xu, F. and Jin, Y.-Q. Target Classification Using the Deep Convolutional Networks for SAR Images. IEEE Transactions on Geoscience and Remote Sensing, 54, 8 (2016), 4806-4817.

[10] Krizhevsky, A., Sutskever, I. and Hinton, G. E. Imagenet classification with deep convolutional neural networks. NIPS, 2012.

[11] Dong, C., Loy, C. C., He, K. and Tang, X. Learning a deep convolutional network for image super-resolution. Springer, 2014.

[12] Zhong, J., Yang, B., Huang, G., Zhong, F. and Chen, Z. Remote sensing image fusion with convolutional neural network. Sensing and Imaging, 17, 1 (2016), 1-16. [13] Radhika, K., Rao, C. and Prasad, V. K. Enhancement of AWiFS Spatial Resolution with SVM Learning. In Proceedings of the 2016 IEEE 6th International Conference on Advanced Computing (IACC) (Bhimavaram, India, (2016). IEEE. [14] Klinkenberg, L. Image Fusion.

[15] Saraf, A. IRS-1C-LISS-III and PAN data fusion: an approach to improve remote sensing based mapping techniques. International fournal of Remote Sensing, 20, 10 (1999), 1929-1934.
[16] Saroglu, E., Bektas, F., Musaoglu, N. and Goksel, C. Fusion of multisensor remote sensing data: assessing the quality of resulting images. Int. Arch. Photogram. Rem. Sens. Spatial. Inform. Sci, 35 (2004), 575-579.

[17] Venkataraman, G., Mahato, B. C., Ravi, S., Rao, Y. and Mathur, P. Fusion of Optical and Microwave Remote Sensing data for Snow Cover Mapping. In Proceedings of the Geoscience and Remote Sensing Symposium, 2004. IGARSS'04. Proceedings. 2004 IEEE International (Anchorage, AK, 2004). IEEE.

[18] Pal, S., Majumdar, T. and Bhattacharya, A. K. ERS-2 SAR and IRS-1C LISS III data fusion: A PCA approach to improve remote sensing based geological interpretation. ISPRS journal of photogrammetry and remote sensing, 61, 5 (2007), 281-297.

[19] Harris, J. R., Murray, R. and Hirose, T. IHS transform for the integration of radar imagery with other remotely sensed data. Photogrammetric Engineering and Remote Sensing, 56, 12 (1990), 1631-1641.

[20] Yonghong, J. Fusion of landsat TM and SAR image based on principal component analysis. Remote Sensing Technology and Application, 13, 1 (1998), 46-49. [21] Zhou, J., Civco, D. and Silander, J. A wavelet transform method to merge Landsat TM and SPOT panchromatic data. International fournal of Remote Sensing, 19, 4 (1998), 743-757.

[22] Huang, D. and Yang, M. SAR and Multi-spectral images fusion based on Contourlet and HIS Transform. In Proceedings of the Wireless Communications Networking and Mobile Computing (WiCOM), 2010 6th International Conference on (Chengdu, China, 2010). IEEE.

[23] Bannari, A., El-Battay, A., Saquaque, A. and Miri, A. PALSAR-FBS L-HH Mode and Landsat-TM Data Fusion for Geological Mapping. Advances in Remote Sensing, 5,04 (2016), 246

[24] Rao, C., Malleswara Rao, J., Senthil Kumar, A. and Dadhwal, V. Fast spatiotemporal data fusion: merging LISS III with AWiFS sensor data. International fournal of Remote Sensing, 35, 24 (2014), 8323-8344.

[25] Li, S., Yin, H. and Fang, L. Remote sensing image fusion via sparse representations over learned dictionaries. IEEE Transactions on Geoscience and Remote Sensing, 51, 9 (2013), 4779-4789.

[26] Rao, C., Malleswara Rao, J., Senthil Kumar, A., Lakshmi, B. and Dadhwal, V. Expansion of LISS III swath using AWiFS wider swath data and contourlet coefficients learning. GIScience \& Remote Sensing, 52, 1 (2015), 78-93.

[27] Patwary, M. A. A., Champati ray, P. K. and Parvaiz, I. IRS-LISS-III and PAN data analysis for landslide susceptibility mapping using heuristic approach in active tectonic region of Himalaya. Fournal of the Indian Society of Remote Sensing, 37, 3 (2009), 493-509.

[28] Dong, C., Loy, C. C. and Tang, X. Accelerating the super-resolution convolutional neural network. In Proceedings of the European Conference on Computer Vision (Amsterdam, The Netherlands, 2016). Springer.

[29] Kim, J., Kwon Lee, J. and Mu Lee, K. Accurate image super-resolution using very deep convolutional networks. In Proceedings of the Proceedings of the IEEE Conference on Computer Vision and Pattern Recognition (Seattle, WA, 2016).

[30] Wang, L., Lin, Z., Gao, J., Deng, X. and An, W. Fast single image superresolution based on sigmoid transformation. arXiv preprint arXiv:1708.07029 (2017) [31] Ledig, C., Theis, L., Huszár, F., Caballero, J., Cunningham, A., Acosta, A., Aitken, A., Tejani, A., Totz, J. and Wang, Z. Photo-realistic single image superresolution using a generative adversarial network. arXiv preprint arXiv:1609.04802 (2016).

[32] Masi, G., Cozzolino, D., Verdoliva, L. and Scarpa, G. Pansharpening by convolutional neural networks. Remote Sensing, 8, 7 (2016), 594.

[33] Palsson, F., Sveinsson, J. R. and Ulfarsson, M. O. Multispectral and Hyperspectral Image Fusion Using a 3-D-Convolutional Neural Network. IEEE Geoscience and Remote Sensing Letters, 14, 5 (2017), 639-643.

[34] Seshadri, K., Rao, M., Jayaraman, V., Thyagarajan, K. and Murthi, K. S. Resourcesat-1: A global multi-observation mission for resources monitoring. Acta Astronautica, 57, 2 (2005), 534-539.

[35] Pandya, M., Murali, K. and Kirankumar, A. Quantification and comparison of spectral characteristics of sensors on board Resourcesat-1 and Resourcesat-2 satellites. Remote sensing letters, 4, 3 (2013), 306-314.

[36] Clevert, D.-A., Unterthiner, T. and Hochreiter, S. Fast and accurate deep network learning by exponential linear units (elus). arXiv preprint arXiv:1511.07289 (2015).

[37] Wang, Z., Bovik, A. C., Sheikh, H. R. and Simoncelli, E. P. Image quality assessment: from error visibility to structural similarity. IEEE transactions on image processing, 13, 4 (2004), 600-612.

[38] Huynh-Thu, Q. and Ghanbari, M. Scope of validity of PSNR in image/video quality assessment. Electronics letters, 44, 13 (2008), 800-801.

[39] GDAL Development Team GDAL - Geospatial Data Abstraction Library, Version 1.10. Open Source Geospatial Foundation, 2017.

[40] Kingma, D. and Ba, J. Adam: A method for stochastic optimization. arXiv preprint arXiv:1412.6980 (2014). 